\title{
On the causes of the production of atmospheric electricity in general, in storms, and in heat lightning
}

\section{M. de Tromelin}

To cite this article: $M$. de Tromelin (1884) On the causes of the production of atmospheric electricity in general, in storms, and in heat lightning, Philosophical Magazine Series 5, 17:105, 245-247, DOI: $10.1080 / 14786448408627509$

To link to this article: http://dx.doi.org/10.1080/14786448408627509

曲 Published online: 29 Apr 2009.

Submit your article to this journal $\square$

Џll Article views: 2

Q View related articles ๘ 
ELEVATION OF LAND UURIXG THF GLACIAL PERIOD.

[We have been requested by Prof. Dana to insert the following Note in order to correct any wrong impression that might possibly be formed by the reader from the statement of Dr. Croll on page 13 of our last Number.--EDs.]

The opinion advocated formerly, and now, by Mr. Dana is that the more nortbern lands (or, in later papers, porions of northern lands) were more elevated than now during the era of increasing and maximum ice, or the era distinguished by him as the Glacial period ; that this era was followed, both in America and Europe, by a subsidence of the same land initiating the Champlain period, and that this was the era of melting, and of the spreading of mammals northward, an impossible occurrence in America during the Glacial era; that another era of ice of much less extent occurred subsequently in Europe, if not also in North America, probably commencing with the epoch of the change in the land to its present level, and that this was the occasion of the destruction of the mammals of North Siberia, and other faunal changes. The evidences believed to favour these conclusions are stated in his various papers and his 'Manual of Geology,' and need not be here repeated. The latest discussion by him of the facts from Eastern North America as to the Champlain subsidence is contained in the 'American Journal of Science' for 1882 .

Mr. Dana's opinion as to the fact of an elevation of northern lands in the Glacial era (that is, the era as he defines it) was a conclusion from facts that had been observed in Europe and America, and not a supposition suggested by, or thought to be sustained by, any theory as to the cause of the elevation. The era of maximum ice he bas always supposed to be that of maximum or nearly maximum cold ; and the Champlain era, following, an interglacial era (for Europe at least) of milder climate, in which the Mammoth and the associated mammals and other species of life, animal and vegetable, of the colder temperate and temperate latitudes, reached their furthest northern limit.

ON THE CAUSES OF THE PRODUCTION OF ATMOSPHERIC ELECTRICITY IN GENERAL, IN STORMS, AND IN HEAT LIGHTNING. BY M. DE TROMELIN.

In seeking in nature for causes capable of producing electricity, I have been led to assume in the first case the friction of moist or dry air against the surface of the earth or of the seas.

Every one knows A rmstrong's hydro-electrical machine. When the steam energes from the boiler, the latter is charged negatively 
while the steam is positive. In the machine there is a box full of water to cool the tubes through which the steam emerges. Hence before the steam escapes it begins to condense, and thus passes out mixed with vesicles of water. This is a necessary condition.

According to Faraday's experiments the passage of dry steam or of a current of dry air does not liberate electricity, while a current of moist air gives the same result as Armstrong's machine, but to a less extent. M. Spring, it is true, found that the friction of dry air against a copper sphere gave a little electricity, but a quantity $f a$. less than in Faraday's experiment. On the other hand, it has been shown by numerous experiments that the mere evaporation of water, even when acidulated, does not produce electricity. But in evaporation produced by wind there is friction, and in this mechanical work the desired cause is to be sought.

The wind in grazing the surface of the sea carries with it the aqueous particles of the crest of the waves, which here play the part of the comb in Armstrong's machine. The roughnesses on the surface of the soil play the same part when a moist wind blows over them. These particles of water retain their electricity, and rising in the atmosphere form clouds, and as electricity passes to the surface of bodies, they tend to spread over the highest atmospheric surfaces; and I hold, with M. Faye, that the cirrus must retain great part of this electricity.

That being admitted, it may be conceived that a cloud is electrified if formed in the conditions just cited. It would not be the case with a cloud formed by mere vapours rising in the air in the morning.

But to produce lightning there must be a discharge between the cloud and another point, the earth or cloud, such that the difference of potential between the point and the cloud, at the moment in question, is sufficient to produce the lightning.

But the distance across which the spark passes depends on the electric pressure and on the mechanical resistance which the medium presents to the discharge. This pressure varies with the square of the potential of the cloud, its form, its surface, the charge of the cloud, and its distance from the point in question.

But the potential energy of a cloud depends on its form, its surface, and its temperature. When this cloud originally electrified undergoes any change whatever (condensation, congelation of aqueous vesicles, \&c.), it absorbs a certain amount of energy, which should be found in the form of an increase of potential energy.

This consequence results from the principle of the conservation of energy, and from Carnot's principle, from which Lippmann bas deduced such remarkable results.

If, then, we suppose that the charge of the cloud remains constant for a certain time, condensation or lowering of temperature will increase the potential of the mass.

During a storm, it is very exceptional when there are no electrical manifestations. The abundant condensation then observed is 
the cause of an increase of potential of the storm-cloud, and when this potential is sufficient, discharge takes place in the form of lightning. Moreover, the continual renewal of the charge indicates that the cause must reside in the storms themselves.

Air being a dielectric, the vapour becomes the natural reservoir of the electricity. Imagine a mass of electrified aqueous vapour in suspension in the air: at night, when the sun has disappeared, there will be a cooling of the cloud-mass. For the reason above given its potential will increase.

Now if the potential reaches a certain extent, the vesicles considered separately can no longer retain their charge-that is to say, they will escape into the air in the form of silent discharges.

It is calculated that, at the ordinary pressure, a small sphere, 0.6 millim. in diameter, cannot retain electricity at the potential obtained with our ordinary machines.

From the small radius of the vesicles it will be seen that a cloud cannot retain a charge at a potential exceeding a certain amount. In this way would be produced the phenomenon known as heat lightring.

As these lightning flashes are seen by different observers in the horizon, it is logical to suppose that they are also produced at the zenith, and that they are not the reflections of the lightning due to storms at a distance. A similar phenomenon occurs with a traveller who always sees a laver of fog towards the horizon, while the sky above is clear. This is only due to a different thickness of the layers traversed by the visual rays.-Comptes Rendus, Jan. 28, 1884.

\section{NOTE ON THE TEMPERATURE OF SUN-SPOTS. BY PROF. EILEARD WIEDEMANN*.}

Messrs. Liveing and Dewar, in one part of their very interesting paper (Phil. Mag. vol. xvi. p. 402), have endeavoured to show that the temperature of the sun-spots may be higher than that of the surrounding surface of the sun, although their thermal and chemical effects are much less. The authors are of the opinion that the intensity of the total radiation from the spots may be much larger than that from the rest of the surface, containing a greater proportion of violet and ultra-violet rays which are absorbed at the border of our atmosphere. In these conclusions I cannot agree with them. In increasing the temperature of a luminous source it is true that the intensity of the more-refrangible part of the spectrum increases more rapidly than that of the less-refrangible part; but, nevertheless, the intensity of the latter does increase; so that, of two sources of different temperature, the botter emits in every part of the spectrum more light than the colder, at least as long as the spectrum is continuous.

* Communicated by the Author. 